\title{
DUAL EXTREMUM PRINCIPLES FOR HEAT-TRANSFER PROBLEMS WITH VARIABLE THERMAL PROPERTIES*
}

\author{
BY \\ N. ANDERSON AND A. M. ARTHURS \\ University of York
}

1. Introduction. In a recent paper, Aziz and Benzies [1] used regular perturbation techniques to obtain approximate solutions of some heat-transfer problems with temperature-dependent thermal properties. In the present paper, we adopt a different approach which is based on the theory of dual extremum principles [cf. 2, 3]. These principles provide the basis of variational methods which lead to variational solutions of known accuracy. The results are illustrated by two applications, one concerning a conductingconvecting straight fin with variable heat-transfer coefficient, and the other concerning the temperature distribution in a conducting-convecting-radiating fin with temperature-dependent thermal conductivity. In both cases very accurate variational solutions are obtained.

2. A class of problems. The problems we wish to consider are described by equations of the form

$$
-d^{2} \phi / d x^{2}=f(\phi), \quad 0<x<1,
$$

with boundary conditions

$$
\phi^{\prime}(0)=0, \quad \phi(1)=k .
$$

Here $k$ is a prescribed constant and $f(\phi)$ is a known differentiable function of $\phi$. In the applications, $\phi$ will denote the required nondimensional temperature.

To use the theory of dual extremum principles we first rewrite Eqs. (1) and (2) in canonical form by taking

$$
\begin{aligned}
d \phi / d x & =u=\partial H / \partial u, \\
-d u / d x=f(\phi) & =\partial H / \partial \phi, \quad 0<x<1,
\end{aligned}
$$

with

$$
\begin{aligned}
& \phi(1)=k, \\
& u(0)=0 .
\end{aligned}
$$

A suitable Hamiltonian $H$ in Eqs. (3) and (4) is given by

$$
H(u, \phi)=\frac{1}{2} u^{2}+F(\phi),
$$

* Received June 14, 1977. 
where

$$
F(\phi)=\int^{\phi} f(v) d v
$$

The canonical variables $u, \phi$ used here are analogous to the momentum and position $p, q$ variables in conventional Hamiltonian mechanics.

3. Variational principles. To give a variational formulation of Eqs. (3) to (6) we introduce the action functional

$$
\begin{aligned}
I(U, \Phi) & =\int_{0}^{1}\left\{-U \Phi^{\prime}-H(U, \Phi)\right\} d x-\{U(\Phi-k)\}_{x=1}, \\
& =\int_{0}^{1}\left\{-U^{\prime} \Phi-H(U, \Phi)\right\} d x+U(1) k-U(0) \Phi(0),
\end{aligned}
$$

where $H(U, \Phi)$ is defined by Eq. (7). It then follows that

3(a). Variational principle: For arbitrary independent functions $U, \Phi$, the functional $I(U, \Phi)$ is stationary at the solution $(u, \phi)$ of the boundary-value problem described by Eqs. (3) to (6).

Next we consider extremum principles, that is, maximum and minimum principles, associated with our class of boundary-value problems.

3(b). First extremum principle: Using Eq. (9a) we define a functional $J\left(\phi_{1}\right)$ by

$$
J\left(\phi_{1}\right)=I\left(u_{1}, \phi_{1}\right)
$$

where $u_{1}$ and $\phi_{1}$ satisfy

$$
d \phi_{1} / d x=u_{1}, \quad \phi_{1}(1)=k
$$

This gives

$$
\begin{aligned}
J\left(\phi_{1}\right) & =\int_{0}^{1}\left\{\frac{1}{2}\left(\phi_{1}^{\prime}\right)^{2}-F\left(\phi_{1}\right)\right\} d x, \quad \phi_{1}(1)=k \\
& =I(u, \phi)+\Delta J,
\end{aligned}
$$

where

$$
\Delta J=\frac{1}{2} \int_{0}^{1}\left\{\left(\phi_{1}{ }^{\prime}-\phi^{\prime}\right)^{2}-\left(\phi_{1}-\phi\right)^{2} \frac{\overline{d f}}{d \phi}\right\} d x,
$$

the bar over a derivative indicating that it is to be evaluated for some function $\phi+\eta\left(\phi_{1}\right.$ $\phi), 0<\eta<1$. Eq. (13) shows that $J\left(\phi_{1}\right)$ is stationary at $\phi$. This is the Euler-Lagrange variational principle for the original problem described by Eqs. (1) and (2). Further, if

$$
\frac{d f(v)}{d v} \leq 0 \text { in } 0<x<1 \text { for all } v
$$

we see from Eq. (14) that

$$
\Delta J \geq 0 \text { for all } \phi_{1},
$$

and by (13) we therefore have the global minimum principle 


$$
I(u \phi) \leq J\left(\phi_{1}\right)
$$

for all admissible functions $\phi_{1}$ satisfying the condition $\phi_{1}(1)=k$.

3(c). Second extremum principle: Next, using Eq. (9b), we define a functional $G\left(u_{2}\right)$ by

$$
G\left(u_{2}\right)=I\left(u_{2}, \phi_{2}\right)
$$

where $u_{2}$ and $\phi_{2}$ satisfy

$$
-\left(d u_{2} / d x\right)=f\left(\phi_{2}\right), \quad 0<x<1, \quad u_{2}(0)=0 .
$$

This gives

$$
\begin{aligned}
G\left(u_{2}\right) & =\int_{0}^{1}\left\{-u_{2} f^{-1}\left(-u_{2}{ }^{\prime}\right)-\frac{1}{2} u_{2}^{2}-F\left[f^{-1}\left(-u_{2}{ }^{\prime}\right)\right]\right\} d x+k u_{2}(1), u_{2}(0)=0, \\
& =I(u, \phi)+\Delta G,
\end{aligned}
$$

where

$$
\Delta G=-\frac{1}{2} \int_{0}^{1}\left\{\left(u_{2}-u\right)^{2}-\left(f^{-1}\left(-u_{2}^{\prime}\right)-\phi\right)^{2} \frac{\overline{d f}}{d \phi}\right\} d x
$$

the bar over a derivative this time indicating that it is to be evaluated at some function $u+$ $\eta\left(u_{2}-u\right), 0<\eta<1$. Eq. (21) shows that $G\left(u_{2}\right)$ is stationary at $u$. In addition, if the condition

$$
d f / d v \leq 0 \text { in } 0<x<1, \text { for all } v,
$$

is satisfied, we see from Eq. (22) that

$$
\Delta G \leq 0 \text { for all } u_{2},
$$

and so by Eq. (21) we have the global maximum principle

$$
G\left(u_{2}\right)<I(u, \phi)
$$

for all admissible functions $u_{2}$ satisfying the condition $u_{2}(0)=0$.

3(d). Dual extremum principles: From the results in 3(b) and 3(c) we have the global dual extremum principles

$$
G\left(u_{2}\right) \leq I(u, \phi) \leq J\left(\phi_{1}\right)
$$

in the case when

$$
d f(v) / d v \leq 0 \text { for all } v,
$$

for all admissible functions $\phi_{1}$ and $u_{2}$ such that

$$
\phi_{1}(1)=k, \quad u_{2}(0)=0 .
$$

Equality holds in Eq. (25) when $\phi_{1}=\phi$ and $u_{2}=u$.

4. Error estimate. The dual extremum principles in $3(d)$ enable us to obtain an estimate of the error in the variational function $\phi_{1}$. Thus from (25) we have 


$$
\begin{aligned}
\Delta & \equiv J\left(\phi_{1}\right)-G\left(u_{2}\right) \geq J\left(\phi_{1}\right)-I(u, \phi) \\
& =\frac{1}{2} \int_{0}^{1}\left\{\left(\phi_{1}^{\prime}-\phi^{\prime}\right)^{2}-\left(\phi_{1}-\phi\right)^{2} \frac{\overline{d f}}{d \phi}\right\} d x,
\end{aligned}
$$

by (14). By Eq. (26), which we suppose is satisfied, Eq. (28) gives

$$
\begin{aligned}
2 \Delta & \geq \int_{0}^{1}\left(\phi_{1}^{\prime}-\phi^{\prime}\right)^{2} d x \\
& =\int_{0}^{1}\left(\phi_{1}-\phi\right)\left(-\frac{d^{2}}{d x^{2}}\right)\left(\phi_{1}-\phi\right) d x+\left[\left(\phi_{1}-\phi\right)\left(\phi_{1}{ }^{\prime}-\phi^{\prime}\right)\right]_{0}{ }^{1}
\end{aligned}
$$

To obtain a useful result from this we make the boundary terms vanish. This is done by imposing the extra condition

$$
\phi_{1}^{\prime}(0)=0
$$

on $\phi_{1}$, in addition to the condition $\phi_{1}(1)=k$ already required for dual principles. Then Eq. (29) becomes

$$
\begin{aligned}
2 \Delta & \geq \int_{0}^{1}\left(\phi_{1}-\phi\right)\left(-\frac{d^{2}}{d x^{2}}\right)\left(\phi_{1}-\phi\right) d x \\
& \geq \Lambda \int_{0}^{1}\left(\phi_{1}-\phi\right)^{2} d x
\end{aligned}
$$

where $\Lambda$ is a lower bound to the lowest eigenvalue $\lambda_{0}$ of

$$
-\frac{d^{2} w}{d x^{2}}=\lambda w, \quad 0<x<1, \quad w^{\prime}(0)=0, \quad w(1)=0 .
$$

We find that $\lambda_{0}=\pi^{2} / 4$, and so we can take

$$
\Lambda=\pi^{2} / 4 \text {. }
$$

Hence

$$
\int_{0}^{1}\left(\phi_{1}-\phi\right)^{2} d x \leq \frac{8 \Delta}{\pi^{2}}=\frac{8}{\pi^{2}}\left\{J\left(\phi_{1}\right)-G\left(u_{2}\right)\right\} \equiv E\left(\phi_{1}\right),
$$

say. $E$ provides a mean square estimate of the error in the variational function $\phi_{1}$.

5. Problem I. We now turn to applications of the foregoing variational results, and our first application is concerned with a conducting-convecting straight fin with variable heat-transfer coefficient. The problem is described by the nonlinear differential equation [1]

$$
d^{2} \phi / d x^{2}=N^{2} \phi^{1+\epsilon}, \quad 0<x<1,
$$

with

$$
\phi^{\prime}(0)=0, \quad \phi(1)=1 .
$$

Here $N$ is the fin parameter, $\epsilon>0$ is a perturbation parameter, and $\phi$ is the nondimensional temperature distribution. 
Problem I corresponds to the choices

$$
f(v)=-N^{2} v^{1+\epsilon}, \quad k=1,
$$

in the general theory. From (37) we see that

$$
d f / d v=-N^{2}(1+\epsilon) v^{\epsilon}
$$

and this is certainly nonpositive for all $v \geq 0$. We can therefore use the results of $3(d)$ and obtain global dual extremum principles. From Eqs. (12) and (20) we find that the basic functionals $J$ and $G$ are given by

$$
J\left(\phi_{1}\right)=\int_{0}^{1}\left\{\frac{1}{2}\left(\phi_{1}{ }^{\prime}\right)^{2}+\frac{N^{2}}{1+\epsilon}{\phi_{1}}^{2+\epsilon}\right\} d x, \quad \phi_{1}(1)=1,
$$

and

$$
G\left(u_{2}\right)=-\int_{0}^{1}\left\{\frac{1}{2} u_{2}^{2}+\frac{1+\epsilon}{2+\epsilon} N^{2}\left(\frac{u_{2}^{\prime}}{N^{2}}\right)^{(2+\epsilon) /(1+\epsilon)}\right\} d x+u_{2}(1), \quad u_{2}(0)=0 .
$$

It follows from the theory of Sec. 3 that the global dual extremum principles

$$
G\left(u_{2}\right) \leq I(u, \phi) \leq J\left(\phi_{1}\right)
$$

hold, provided the trial functions for temperature are nonnegative.

6. Calculations for problem I. We have performed calculations with trial functions of the form

$$
\begin{aligned}
& \phi_{1}=1+\sum_{n=2}^{6} a_{n}\left(x^{n}-1\right), \\
& u_{2}=\sum_{n=1}^{6} b_{n} x^{n}
\end{aligned}
$$

which satisfy the essential conditions $\phi_{1}(1)=1, u_{2}(0)=0$, and the error estimate condition $\phi_{1}{ }^{\prime}(0)=0$. The coefficients $a_{n}$ and $b_{n}$ were determined by optimizing the functionals $J$ and $G$, and the results for $N=1, \epsilon=0.25$ are given in Table 1 . Since the mean-square error in $\phi_{1}$ is less than $E=6 \times 10^{-6}$, the variational solution $\phi_{1}$ in Eq. (42) is very accurate.

7. Problem II. Our second application of the variational theory concerns the prob-

\begin{tabular}{|c|c|c|c|c|c|c|c|}
\hline $\begin{array}{c}a_{2} \\
0.25190\end{array}$ & $\begin{array}{c}a_{3} \\
0.16269\end{array}$ & $\begin{array}{c}a_{4} \\
-0.11734\end{array}$ & $\begin{array}{c}a_{5} \\
-0.01002\end{array}$ & $\begin{array}{c}a_{6} \\
0.04415\end{array}$ & & $\begin{array}{c}J \\
0.332194\end{array}$ & \\
\hline $\begin{array}{c}b_{1} \\
0.59917\end{array}$ & $\begin{array}{c}b_{2} \\
0.03315\end{array}$ & $\begin{array}{c}b_{3} \\
0.03838\end{array}$ & $\begin{array}{c}b_{4} \\
0.05957\end{array}$ & $\begin{array}{c}b_{5} \\
0.00868\end{array}$ & $\begin{array}{c}b_{6} \\
-0.01068\end{array}$ & $\begin{array}{c}G \\
0.332187\end{array}$ & $\begin{array}{c}E \\
0.000006\end{array}$ \\
\hline
\end{tabular}
lem of the temperature distribution in a conducting-convecting-radiating fin with temperature-dependent thermal conductivity. The problem is described by the nonlinear differential equation [1]

$$
\frac{d}{d x}\left\{\left(1+\epsilon_{1} \theta\right) \frac{d \theta}{d x}\right\}=N^{2} \theta+\epsilon_{2} \theta^{4}, \quad 0<x<1
$$

TABLE 1. Variational parameters for problem I with $N=1, \epsilon=0.25$. 
with

$$
\theta^{\prime}(0)=0, \quad \theta(1)=1 .
$$

Here $\epsilon_{1}$ and $\epsilon_{2}$ are nonnegative perturbation parameters, and $\theta$ is the temperature distribution. To put these equations in a suitable form we set

$$
\phi=\theta+\frac{1}{2} \epsilon_{1} \theta^{2},
$$

for then the problem becomes

$$
d^{2} \phi / d x^{2}=N^{2} a(\phi)+\epsilon_{2} a^{4}(\phi), \quad 0<x<1,
$$

with

$$
\phi^{\prime}(0)=0, \quad \phi(1)=1+\frac{1}{2} \epsilon_{1},
$$

where

$$
a(\phi)=\frac{1}{\epsilon_{1}}\left\{-1+\left(1+2 \epsilon_{1} \phi\right)^{1 / 2}\right\} .
$$

From Eq. (46),

$$
\theta=a(\phi)
$$

enables us to recover the original function $\theta$ from the secondary function $\phi$.

Problem II described by Eqs. (47) and (48) corresponds to the choices

$$
\begin{gathered}
f(v)=-N^{2} a(v)-\epsilon_{2} a^{4}(v), \\
k=1+\frac{1}{2} \epsilon_{1}
\end{gathered}
$$

in the general theory. From (51) it follows that

$$
d f(v) / d v \leq 0 \text { for all } v \geq 0 .
$$

We can therefore use the results of Sec. 3 and obtain global dual extremum principles. From Eqs. (12) and (20) we find that the basic functionals $J$ and $G$ are

$$
J\left(\phi_{1}\right)=\int_{0}^{1}\left\{\frac{1}{2}\left(\phi_{1}{ }^{\prime}\right)^{2}-F\left(\phi_{1}\right)\right\} d x, \quad \phi_{1}(1)=1+\frac{1}{2} \epsilon_{1},
$$

and

$$
\begin{aligned}
G\left(u_{2}\right)= & \int_{0}^{1}\left\{-u_{2}^{\prime} f^{-1}\left(-u_{2}^{\prime}\right)-\frac{1}{2} u_{2}{ }^{2}-F\left[f^{-1}\left(-u_{2}^{\prime}\right)\right]\right\} d x \\
& +\left(1+\frac{1}{2} \epsilon_{1}\right) u_{2}(1), \quad u_{2}(0)=0 .
\end{aligned}
$$

It follows from the theory of Sec. 3 that the global dual extremum principles

$$
G\left(u_{2}\right) \leq I(u, \phi) \leq J\left(\phi_{1}\right)
$$

hold for nonnegative trial functions for temperature.

8. Calculations for problem II. We have performed calculations with trial functions of the form 
TABLE 2. Variational parameters for problem II with $N=1, \epsilon_{1}=\epsilon_{2}=0.2$.

\begin{tabular}{cccccccc}
\hline$a_{2}$ & $a_{3}$ & $a_{4}$ & $a_{5}$ & $a_{6}$ & & $J$ \\
0.35975 & 0.01675 & -0.04888 & 0.08738 & -0.02575 & & 0.457523 & \\
$b_{1}$ & $b_{2}$ & $b_{3}$ & $b_{4}$ & $b_{5}$ & $b_{6}$ & $G$ & $E$ \\
0.70210 & 0.01920 & 0.12570 & -0.06450 & 0.09270 & -0.02050 & 0.457521 & 0.000002 \\
\hline$\phi_{1}$ & $=1+\frac{1}{2} \epsilon_{1}+\sum_{n=2}^{6} a_{n}\left(x^{n}-1\right)$, \\
$u_{2}$ & $=\sum_{n=1}^{6} b_{n} x^{n}$,
\end{tabular}

which satisfy the essential conditions $\phi_{1}(1)=1+\frac{1}{2} \epsilon_{1}, u_{2}(0)=0$, and the error estimate condition $\phi_{1}{ }^{\prime}(0)=0$. The coefficients $a_{n}$ and $b_{n}$ were determined by optimizing the functionals $J$ and $G$ in (54) and (55), and the results for $N=1, \epsilon_{1}=0.2, \epsilon_{2}=0.2$ are given in Table 2. Since the mean-square error in $\phi_{1}$ is less than $E=2 \times 10^{-6}$, the variational solution $\phi_{1}$ in Eq. (57) is very accurate. By (50),

$$
\theta_{1}=a\left(\phi_{1}\right)=\frac{1}{\epsilon_{1}}\left\{-1+\left(1+2 \epsilon_{1} \phi_{1}\right)^{1 / 2}\right\}
$$

is the corresponding variational approximation to the required temperature distribution $\theta$.

9. Conclusion. The solutions for these two problems have shown that variational methods based on dual extremum principles can be usefully employed to treat nonlinear heat transfer problems. The error estimates were found to be of the order of $10^{-6}$, indicating great accuracy in the variational solutions.

One advantage of the variational approach is that it is not restricted, as the perturbation method is, to small values of certain parameters.

\section{REFERENCES}

[1] A. Aziz and J. Y. Benzies, Application of perturbation techniques to heat-transfer problems with variable thermal properties, Int. J. Heat Mass Transfer 19, 271-275 (1976)

[2] A. M. Arthurs, Complementary variational principles, Clarendon Press, Oxford (1970)

[3] B. Noble and M. J. Sewell, On dual extremum principles in applied mathematics, J. Inst. Math. Appl. 9, 123-193 (1972) 